\title{
Aortic root endocarditis: a Biointegral Bioconduit subannular implantation
}

\author{
Marco Di Eusanio, Paolo Berretta, Jacopo Alfonsi, Mariano Cefarelli \\ Department of Cardiac Surgery, Lancisi Cardiovascular Center, Polytechnic University of Marche, Ancona, Italy \\ Correspondence to: Prof. Marco Di Eusanio. Department of Cardiac Surgery, Lancisi Cardiovascular Center, Polytechnic University of Marche, \\ Ancona, Italy. Email: m.dieusanio@univpm.it.
}

Submitted Jul 22, 2019. Accepted for publication Aug 22, 2019.

doi: 10.21037/acs.2019.09.02

View this article at: http://dx.doi.org/10.21037/acs.2019.09.02

\section{Introduction}

Infective endocarditis (IE) with extensive annular disruption and aortic root involvement carries an ominous prognosis $(1,2)$. The broad and severe impairment of the peri-annular tissues at the level of the interventricular septum and mitro-aortic continuity may hamper a successful surgical reconstruction, with dehiscence and retraction of the anterior mitral leaflet (AML) being a possible dreadful complication $(3,4)$. Here we present our technique for root replacement (Bentall) that involves a sub-annular implantation of a $100 \%$ pericardial valved conduit (Bioconduit ${ }^{\mathrm{TM}}$, Biointegral Surgical, Inc., Ontario, Canada).

\section{Clinical vignette}

A 72-year-old male presenting with persistent nocturnal fever and New York Heart Association (NYHA) class III symptoms was referred to our institution. He had undergone mechanical aortic valve replacement and endocavitary definitive pacemaker implantation 12 years earlier. Blood cultures grew Enterococcus faecalis, and the transesophageal echocardiography showed severe periprosthetic aortic regurgitation associated with a large aortic root pseudoaneurysm.

\section{Surgical techniques}

\section{Preparation \& exposition}

The aorta was approached with a median re-sternotomy. Cardiopulmonary bypass was instituted by means of aortic arch and bicaval cannulation, and a left ventricular drain was inserted through the right superior pulmonary vein. Cardioplegic arrest was achieved using retrograde and antegrade cold crystalloid cardioplegia.

\section{Operation}

The aorta was opened $4 \mathrm{~cm}$ above the right coronary ostium, and the aortic root was extensively freed from the surrounding tissue, down to the subvalvular plane, using electrocautery and scissors. The prosthetic aortic valve was removed and the coronary ostia carefully prepared and suspended. A full mobilization of the coronary ostia is recommended to prevent distortion and kinking of the coronaries during reattachment and to reduce the tension of the anastomosis.

The aortic sinuses were resected and an appropriate biological pericardial conduit was selected. The proximal suture line for the Bioconduit implantation runs in a continuous fashion on the left ventricular outflow tract at the robust lower edge of the abscess with bites including the intact muscular septum and only a few millimeters of the AML. As a result, the abscess was kept open and draining into the pericardial cavity. A hole for left coronary button reimplantation was made in the pericardial conduit using a $4 \mathrm{~mm}$ punch. The coronary button was trimmed, keeping a $2-3 \mathrm{~mm}$ aortic cuff, and a parachute anastomosis was performed with a 5-0 (or 6-0) polypropylene running suture. The right coronary artery was then re-attached in the same manner.

\section{Completion}

The distal end of the Bioconduit was shortened to approximate it to the distal end of the aorta. The valve conduit was sutured 
to the distal aorta with a continuous 4-0 polypropylene running suture using parachute technique.

The endocavitary wires and the pacemaker were removed, and after coronary reperfusion, two epicardial leads were implanted on the right atrium and right ventricle. These wires were then connected to a new subcutaneous pacemaker. The intervention was then concluded in the usual fashion.

Total extracorporeal circulation time and crossclamp times were 119 and 100 minutes respectively. The postoperative course was uneventful and the patient was discharged on post-operative day 7 .

\section{Comment}

Root reconstruction in patients presenting with IE with extensive peri-annular abscess represents a great surgical challenge. Graft dehiscence, mitral valve distortion or dysfunction, and tension or kinking at the re-implanted coronary arteries represent the major technical concerns in these patients.

At the Lancisi Cardiovascular Center in Ancona we developed a technique for root replacement that involves a sub-annular implantation of a $100 \%$ pericardial valved conduit. With this very low suture line, we keep the abscess draining outside into the pericardial cavity and deliberately renounce reconstructing the annulus, which may generate tension on the friable anatomical structures, thus causing graft dehiscence. The stentless Bioconduit is extremely pliable and adapts very well to the irregular surfaces of infective pseudoaneurysm; in addition, the absence of the valvular stent reduces mechanical solicitations during the cardiac cycle (5).

Since late 2016, 22 patients (age: 65.6 years) have undergone aortic root replacement due to disruptive endocarditis in our institution. All cases were reoperations and mean EuroSCORE II was 15.8 (range, 6.8-50.4). One patient died due to multi-organ failure; he presented with poor preoperative condition (cardiogenic shock, frontal ischemic lesion, renal failure) with a EuroSCORE II of $45.8 \%$. Three patients required new permanent pacemaker implantation for complete heart block and one experienced transient renal failure requiring dialysis. At a mean follow up of $13 \pm 4$ months all patients were alive with no case of graft reinfection or structural valve deterioration.

In summary, in patients with IE, the presented technique is associated with important advantages including (I) a
$100 \%$ biological root reconstruction, (II) complete abscess exclusion, and (III) no tension on fundamental anatomical structures (septum, AML) likely translating into a reduced risk of suture dehiscence. Despite the high-risk profile of these patients, our preliminary results are promising and suggest safety and efficacy of this approach. However, more robust data and long-term results are still needed.

\section{Acknowledgments}

None.

\section{Footnote}

Conflicts of Interest: The authors have no conflicts of interest to declare.

\section{References}

1. d'Udekem Y, David TE, Feindel CM, et al. Long-term results of surgery for active infective endocarditis. Eur J Cardiothorac Surg 1997;11:46-52.

2. Puehler T, Freitag-Wolf S, Friedrich C, et al. Outcomes of Patients after Implantation of the Pericardial AllBiological Valve No-React Aortic Conduit (BioIntegral) for Root Replacement in Complex Surgical Procedures. Thorac Cardiovasc Surg 2019. [Epub ahead of print].

3. Davierwala PM, Binner C, Subramanian S, et al. Double valve replacement and reconstruction of the intervalvular fibrous body in patients with active infective endocarditis. Eur J Cardiothorac Surg 2014;45:146-52.

4. Uchida T, Kuroda Y, Hamasaki A, et al. Modified Commando operation using stentless aortic bioprosthesis. J Card Surg 2019;34:846-8.

5. Wendt D, Raweh A, Knipp S, et al. Comparison of mid-term haemodynamic performance between the BioValsalva and the BioIntegral valved conduits after aortic root replacement. Interact Cardiovasc Thorac Surg 2016;23:112-7.

Cite this article as: Di Eusanio M, Berretta P, Alfonsi J, Cefarelli M. Aortic root endocarditis: a Biointegral Bioconduit subannular implantation. Ann Cardiothorac Surg 2019;8(6):713-714. doi: 10.21037/acs.2019.09.02 